\title{
The Impact of Medication Reviews Conducted in Primary Care on Hospital Admissions and Mortality: An Observational Follow-Up of a Randomized Controlled Trial
}

This article was published in the following Dove Press journal: Drug, Healthcare and Patient Safety

Veronica Milos Nymberg ${ }^{1,2}$ Cecilia Lenander ${ }^{1,2}$ Beata Borgström Bolmsjö ${ }^{1,2}$

'Department of Clinical Sciences, Lund University, Malmö, Sweden; ${ }^{2}$ CPF, Center for Primary Healthcare Research, Malmö, Sweden
Correspondence: Beata Borgström Bolmsjö

CPF, Center for Primary Health Care Research, Clinical Research Centre (CRC), Building 28, Floor II, Jan Waldenström Street 35, Malmö SE-205 02, Sweden Email beata.borgstrom_bolmsjo@med.lu. se
Background: Drug-related problems among the elderly population are common and increasing. Multi-professional medication reviews (MR) have arisen as a method to optimize drug therapy for frail elderly patients. Research has not yet been able to show conclusive evidence of the effect of MRs on mortality or hospital admissions.

Aim: The aim of this study was to assess the impact of MRs' on hospital admissions and mortality after six and 12 months in a frail population of 369 patients in primary care in a cohort from a randomized controlled study.

Methods: Patients were blindly randomized to an intervention group (receiving MRs) and a control group (receiving usual care). Descriptive data on mortality and hospital admissions at six and 12 months were collected. Survival analysis was performed for time to death and time to the first hospital admission within 12 months.

Results: An observational follow-up was performed in a cohort of 369 patients, previously randomized to an intervention group (182) and a control group (187). Most of the patients (75\%) were females and lived in nursing homes. At six months, 50 patients of the baseline population $(27 \%)$ in the control group had been admitted to hospital at least once, compared to 40 patients $(21 \%)$ in the intervention group. At 12 months, the percentage had increased to $70(37 \%)$ in the control group compared to $53(29 \%)$ in the intervention group. Compared to usual care, we found that MRs reduced the risk of hospital admissions within 12 months by $42 \%(\mathrm{HR}=0.58,95 \%$ CI $0.37-0.92, \mathrm{p}=0.021)$, but found no difference in mortality $(\mathrm{HR}=$ $1.12,95 \%$ CI $0.78-1.61, \mathrm{p}=0.551$ ) between the groups.

Conclusion: We suggest that MRs should be recommended in the care of frail elderly patients with expected benefits on delayed hospital admissions. The study is registered at ClinicalTrials.gov, registration number NCT04040855, Unique Protocol ID 2018/8.

Keywords: medication reviews, primary care, elderly, randomized controlled study, hospital admissions, mortality

\section{Background}

Drug-related problems (DRPs) and polypharmacy in the elderly population are common and increasing. ${ }^{1}$ Lack of a consistent drug list and low physician continuity are some of the reasons for drug-related problems and the need for emergency hospital contacts. ${ }^{1}$ Most of these problems can be avoided and multi-professional medication reviews have arisen as a method to optimize drug therapy for frail elderly 
patients. ${ }^{2,3}$ Meanwhile, medication reviews (MR) have been questioned for their efficacy. ${ }^{4}$ Even if studies have shown that pharmacists' recommendations are clinically relevant, ${ }^{5,6}$ recent research has not been able to demonstrate an effect on mortality or hospital admissions, due to heterogeneity of intervention designs, patient characteristics as well as follow-up time in different studies; hence, the conclusion is that more evidence is needed. ${ }^{7,8}$ Furthermore, a systematic review and meta-analysis of randomized controlled trials with short follow-up time ( $<3$ months) showed no effect on clinical outcomes or quality of life and the researchers argued that it should be considered to stop performing medication reviews as standard care. ${ }^{8}$ This conclusion is controversial as medication reviews are well established in Sweden and Swedish authorities grant incentives and other funding on an annual basis to healthcare providers in order to optimize drug therapy among elderly patients. ${ }^{9}$ It is important to mention that the heterogeneity of study designs and outcomes is a problem while performing meta-analyses.

The model for MRs in primary care in southern Sweden with focus on elderly patients with multiple illnesses is well studied and has been shown to reduce potentially inappropriate medications (PIMs) and consequences due to medication errors. ${ }^{10}$ The integrated approach with a pharmacist helping in the clinical routine had initially been developed in hospital care but has been adapted to primary care. MRs using this approach have now been conducted for the last 15 years, both in nursing homes and community-dwelling elderly patients in Sweden, with the goal to improve quality of medication use and patient safety.

This randomized controlled trial (RCT), which was performed in 2012, included 369 patients and showed that multi-professional medication reviews conducted by teams including nurses, pharmacists and GPs had a lowering effect on the number of drugs as well as on the number of PIMs among elderly patients. ${ }^{11}$ The first results showed that two months after the medication reviews, both the number of patients with at least one PIM and the number of patients having 10 or more drugs had decreased in the intervention group, while there were no statistically significant differences in the control patients. DRPs were identified in $93 \%$ of the 182 patients in the intervention group and $16 \%$ of the DRPs were related to PIMs. The two most common intervention recommendations the pharmacist presented to the physician were withdrawal of drug therapy $(30 \%)$ and reduced dosage (28\%). A significant proportion $(87 \%)$ of the studied population had treatment with fall risk increasing drugs (FRIDs). ${ }^{11}$

As we could show statistically significant benefits in the intervention group receiving MRs in the short followup time, we considered it important to study this further by focusing on mortality data and hospital admissions during a longer follow-up time of six and 12 months.

\section{Aim}

The aim of this study was to assess the impact of multiprofessional MRs on hard outcomes, such as hospital admissions and mortality, at six and 12 months in elderly patients in primary care.

\section{Methods}

\section{Study Setting and Design}

Primary care in Skåne county, southern Sweden is provided by approximately 150 primary healthcare centers (PHCCs), both public and private. All public PHCCs were invited to participate in the study. Four pharmacists with extensive prior experience of performing MRs were recruited to the project and assigned to four different areas in Skåne county. Patients eligible for inclusion were users of the multi-dose drug dispensing system aged 75 years or older, living in nursing homes or community dwelling with municipally provided home care. Patients were included in the study after they provided written consent directly or through relatives in cases of severe cognitive impairment. A total number of 391 were assessed for eligibility and 22 were excluded for the following reasons: not meeting inclusion criteria $(n=5)$, declined to participate $(n=1)$, deceased before data collection at base line $(n=5)$ or other reasons $(\mathrm{n}=11)$.

The study design is observational with a follow-up of the randomized controlled trial with 369 included patients, 182 in the intervention group and 187 in the control group.

\section{Intervention}

The pharmacists randomized patients blindly to the intervention or the control group using non-transparent closed envelopes and with a random number generator, stratified only for geographic area. ${ }^{11}$

All patients had multi-dose drug dispensing, thus ensuring compliance to the drug therapy and the medication changes. The control group received usual care. For patients in the intervention group, the pharmacists 
performed a systematic MR based on a symptom assessment made by a nurse with good knowledge of the patient. The MR consisted of a structured assessment in three steps. ${ }^{3}$ First, a nurse conducted a specific symptom evaluation and health status check including blood pressure, pulse, weight, tendency to fall and confusion and other symptoms using a validated symptom assessment form (Phase-20). ${ }^{11}$ Medication lists were printed by the pharmacists who had received permission to access patients' EMR (electronic medical record) as well as the electronic medication list record. Drug-related problems and PIMs were identified using a structured method. ${ }^{11}$ Based on the symptom assessment form and the medication lists the pharmacists identified and categorised medication problems into nine risk categories: drugs that required therapeutic monitoring; less appropriate drug therapy in the elderly (PIMs); drug not recommended (by the regional drug and therapeutics committee); problems with handling the drugs (eg, swallowing, crushing, inhalation); C/D drug interactions $(\mathrm{C}$ interactions are those involving a drug combination that could require dose adjustment, D interactions are those involving a drug combination that ought to be avoided); drug type or drug dosage not adjusted according to liver/renal function; indication for drug treatment unclear; suboptimal treatment; and drugs causing adverse drug reactions.

PIMs were identified following the national guidelines of the Swedish Board of Health and Welfare on drug therapy in the elderly. ${ }^{12}$

The pharmacists' recommendations were documented in patients' EMRs and communicated to the physician in different ways, depending on the PHCC's routines and organization and consisted of team rounds, written contact, personal contact and telephone contact. The physician decided about medication changes and this was documented in the patient's EMR.

\section{Data Collection}

We followed the intervention and control groups from the initial baseline population of 369 patients for 12 months. For the control group, the follow-up started at the inclusion of the study and for the intervention group, the follow-up started after the first MR.

Primary outcome variables were differences in survival time and time to the first hospital admission between the groups at six and 12 months. Secondary outcomes were difference in number of deaths and difference in number of hospital admissions between the groups at six and 12 months. Data from the EMR and the regional patient database were collected using Swedish personal numbers thus assuring $100 \%$ data coverage.

\section{Data Analysis}

Descriptive data for baseline characteristics as well as mortality and hospital admissions were performed. Kaplan-Meier analysis was used to estimate survival function with time to the first hospital admission and time to death as outcome events. Non-parametric Kaplan-Meier was chosen as the exact survival time and time to the first hospital admission for each patient were known. Because the MRs continued as a part of the routine care about once a year or when needed, initiated by the nurse or the physician, some of the patients in the control group received a MR during the follow-up period after the initial 2 months of participation in the RCT and within 12 months of follow-up. Due to deterioration in health status, some control patients might have received several MRs during the 12 months follow-up if needed. We defined this event as a major protocol deviation and chose to perform a per protocol analysis. The patients in the control group receiving MRs during the follow-up period were censored in the survival analysis. Furthermore, we used the Log rank test for equality across strata (ie, control and intervention group). The proportionality assumption was tested (p-value <0.05), which is the main assumption of the Cox proportional hazard model to determine the relationship between covariate and survival time or time to hospital admission and to obtain hazard ratio (HR). Data were analyzed using SPSS version 25.

\section{Results}

Of the 369 included patients, 182 were randomized to the intervention group and 187 to the control group (Figure 1). Most of the patients (75\%) were females and lived in nursing homes. Patient characteristics of the population at baseline are described in Table 1, with no significant observed differences in age, sex, living form and number of continuous drugs between the intervention and control group. At six months, 33 patients in the control group had received at least one MR. At 12 months, another 22 patients (meaning a total number of 55 patients) in the control group had received at least one MR. Patients were censored from the survival analysis at the time for the first MR during the follow-p time of 6 and 12 months.

There was no difference in number of deaths between the groups at six and 12 months (Table 2). The survival analysis 


\section{Randomization}

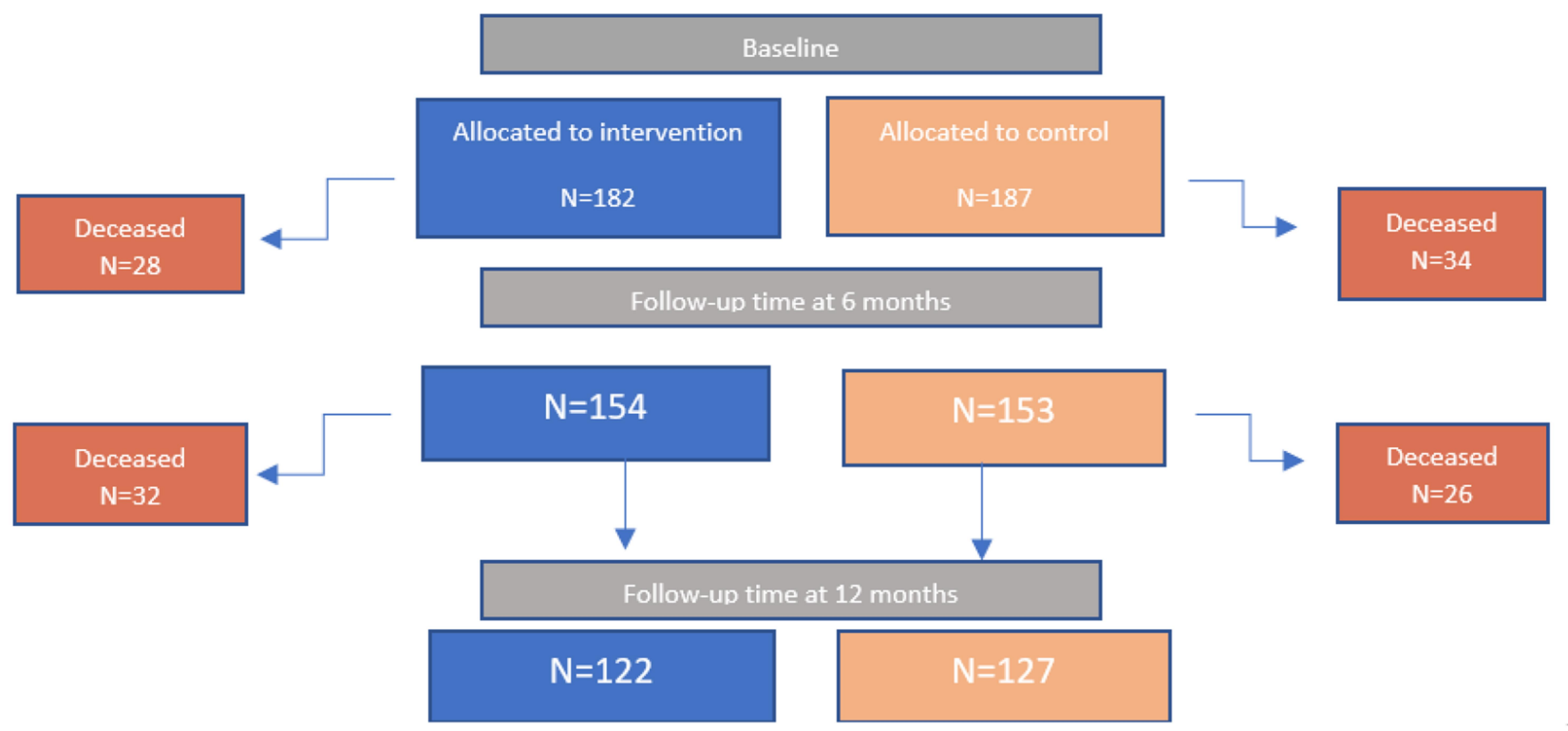

Figure I Flow chart for the studied population at baseline, six and 12 months.

showed no differences in mortality between groups at 12 months ( $\mathrm{p}=0.551)$ with a HR $=1.12(95 \%$ CI $0.78-1.61)$.

At six months, 50 patients of the baseline population (27\%) in the control group had been admitted to hospital at least once, compared to 40 patients $(21 \%)$ in the intervention group. At 12 months the percentage had increased to $70(37 \%)$ in the control group compared to $53(29 \%)$ in the intervention group. However, there was no significant difference between the groups in the number of hospital admissions at 6 months $(\mathrm{p}=0.265)$ or 12 months $(\mathrm{p}=0.092)$ with Mann-Whitney's test. When we excluded patients from the control group that received at least one MR during the follow-up period, after completion of the initial RCT, the difference became significant at 12 months but not at 6 months (Table 3). A graphic comparison between number of hospital admissions between groups at 12 months is presented in Figure 2.
The mean survival time (time to death and time to the first hospital admission) in the groups is presented in Table 4. A survival analysis showed a significant decrease (Log Rank-Mantel Cox $=0.012$ ) in risk for the first hospital admission at 12 months between the intervention and control group (Figure 3). Patients were censored in the analysis at the exact time for event, for death in the intervention group and for death or when receiving a MR in the control group, depending on which event occurred first. A Cox regression analysis was performed to estimate the risk for hospital admissions within 12 months, adjusted for sex, age, number of drugs and number of MRs, with patients censored at the first hospital admission. The Cox regression analysis showed that MRs reduced the risk of hospital admissions by $42 \%$ (HR $=0.58,95 \%$ CI $0.37-0.92)$ compared to usual care $(p=0.021)$. The proportionality assumption was fulfilled.

Table I Population Characteristics at Baseline

\begin{tabular}{|l|l|l|l|l|}
\hline All Patients $\mathrm{n}=369$ & Baseline Characteristics & Intervention $(\mathbf{n}=\mathbf{1 8 2})$ & Control $(\mathbf{n}=\mathbf{1 8 7})$ & $\mathbf{p}$-value \\
\cline { 2 - 5 } & Female sex, $\mathrm{n}(\%)$ & $138(75.8)$ & $142(75.9)$ & $0.980^{*}$ \\
& Institutionalized, $\mathrm{n}(\%)$ & $139(76.4)$ & $140(74.9)$ & $0.809 *$ \\
& Age, mean (SD) & $87.0(5.8)$ & $87.7(5.5)$ & $0.662^{* *}$ \\
& No of continuous drugs (min-max) & $9.2(1-20)$ & $9.7(3-25)$ & $0.528^{* *}$ \\
\hline
\end{tabular}

Notes: ${ }^{*} \mathrm{Chi}^{2}$ test; **Student's $t$-test. 
Table 2 Patient Characteristics and Mortality Numbers at Six and Twelve Months, When Patients in the Control Group Receiving MRs Were Excluded

\begin{tabular}{|c|c|c|c|c|}
\hline \multirow[t]{5}{*}{ At $6 \mathrm{~m}$ (33 Excluded) $\mathrm{n}=336$} & Patient Characteristics & Intervention $(n=\mid 82)$ & Control $(n=154)$ & p-value \\
\hline & Female sex, n (\%) & $138(75.8)$ & $115(74.4)$ & $0.899 *$ \\
\hline & Deceased, n (\%) & $28(15.4)$ & $34(22.0)$ & $0.472 *$ \\
\hline & Institutionalized, n (\%) & $138(76.4)$ & II5 (7I.4) & $0.319 *$ \\
\hline & Age, mean & 87.0 & 87.9 & $0.303^{* *}$ \\
\hline \multirow[t]{5}{*}{ At $12 \mathrm{~m}$ (55 Excluded) $\mathrm{n}=3 \mathrm{I} 4$} & Baseline Characteristics & Intervention $(n=\mid 82)$ & Control $(n=\mid 32)$ & p-value \\
\hline & Female sex, n (\%) & $133(75.1)$ & $103(75.2)$ & $1.000 *$ \\
\hline & Deceased, n (\%) & $60(32)$ & $60(45.4)$ & $0.77 I^{*}$ \\
\hline & Institutionalized, n (\%) & 137 (76.3) & $98(7 \mathrm{I} .5)$ & $0.364^{*}$ \\
\hline & Age (mean) & 87.0 & 87.8 & $0.226 * *$ \\
\hline
\end{tabular}

Notes: ${ }^{*} \mathrm{Chi}^{2}$ test; ${ }^{* * S t u d e n t ' s ~ t-t e s t . ~}$

Table 3 Group Comparison in Hospital Admissions for All Patients and When Patients from Control Group Receiving an MR are Excluded at 6 and 12 Months

\begin{tabular}{|c|c|c|c|c|c|c|}
\hline \multirow{2}{*}{$\begin{array}{l}\text { Population } \\
\text { All patients } \\
n=369\end{array}$} & \multicolumn{3}{|c|}{ Hospital Admissions at 6 Months, (Mean), Min-Max } & \multicolumn{3}{|c|}{ Hospital Admissions at 12 Months, (Mean), Min-Max } \\
\hline & $\begin{array}{l}\text { Intervention } \\
(\mathbf{n}=\mathbf{1 8 2}) \\
(0.29) 0-4\end{array}$ & $\begin{array}{l}\text { Control } \\
(n=187) \\
(0.37) 0-5\end{array}$ & $\begin{array}{l}\text { p-value } \\
0.265^{*}\end{array}$ & $\begin{array}{l}\text { Intervention } \\
(\mathbf{n}=182) \\
(0.45) 0-4\end{array}$ & $\begin{array}{l}\text { Control } \\
(n=187) \\
(0.63) 0-7\end{array}$ & $\begin{array}{l}\text { p-value } \\
0.092^{*}\end{array}$ \\
\hline $\begin{array}{l}\text { Within } 6 \text { m (33 excluded) } \\
n=336\end{array}$ & $\begin{array}{l}\text { Intervention } \\
(\mathbf{n}=\mathbf{1 8 2}) \\
(0.29) 0-4\end{array}$ & $\begin{array}{l}\text { Control } \\
(n=154) \\
(0.40) 0-5\end{array}$ & $\begin{array}{l}\text { p-value } \\
0.180^{*}\end{array}$ & & & \\
\hline $\begin{array}{l}\text { Within } 12 \mathrm{~m} \text { (55 excluded }) \\
\mathrm{n}=314\end{array}$ & & & & $\begin{array}{l}\text { Intervention } \\
(\mathbf{n}=182) \\
(0.46) 0-4\end{array}$ & $\begin{array}{l}\text { Control } \\
(n=132) \\
(0.69) 0-7\end{array}$ & $\begin{array}{l}\text { p-value } \\
0.039 *\end{array}$ \\
\hline
\end{tabular}

Note: *Mann-Whitney's test.

\section{Discussion}

This study found that the patients in the intervention group had a lower risk for first hospital admission within 12 months after the MR compared to the patients in the control group. We found no difference in mortality rate between the groups. The study design with per protocol analysis instead of intention to treat analysis might have contributed to biased survival estimates, as patients in the control group receiving MR during the follow-up time were excluded from the analysis. However, MRs in the control group were performed both as a part of the routine care and due to concerns about medication use that may have been related to condition deterioration. We believe therefore that the risk for bias is lower than expected.

The studied population was elderly individuals that had multimorbidity and polypharmacy. The intervention and the control group were similar concerning age, living form and number of drugs at baseline.
Adverse drug reactions account for a substantial amount of all hospital admissions, ${ }^{13-15}$ and many of these are preventable. ${ }^{14,15}$ Previously published data from this $\mathrm{RCT}^{11}$ showed no difference in number of drugs or PIMs between the groups at baseline but a significantly lower number of drugs and PIMs in the intervention group at two months follow-up, which indicates that MRs might reduce the occurrence of drug-related problems (DRPs). If DRPs can be prevented by reducing the number of drugs and PIMs through MRs, some hospital admissions could be avoided or delayed, and thereby could save both burden and costs for the individual patient as well as for the society.

Many studies of MRs are based on a patient population already admitted to the hospital and have shown that MRs in a hospital setting will extend the time to readmission. ${ }^{16-18}$ A strength of this study was that the MRs were performed in primary care, therefore adding 


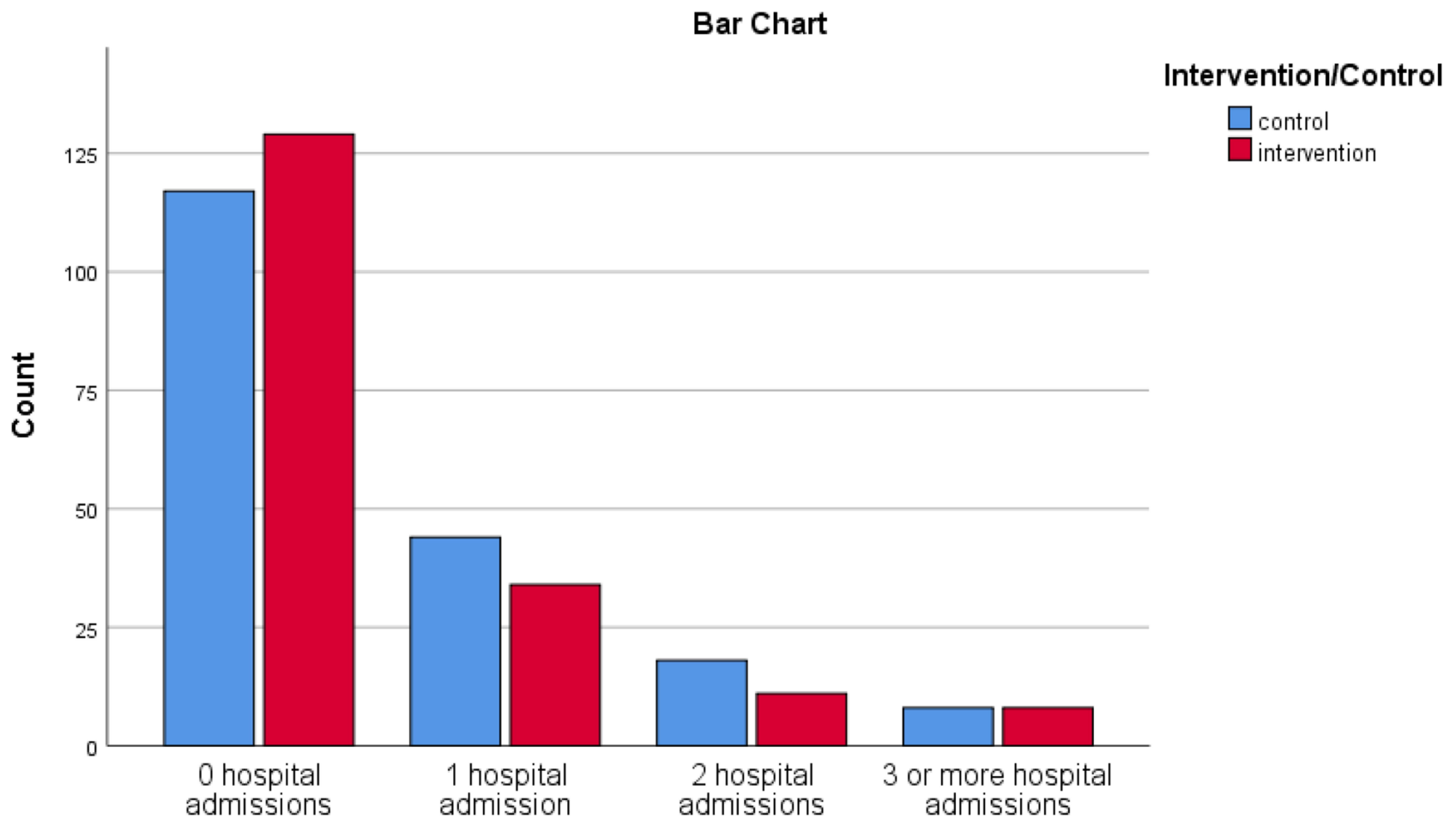

Number of hospital admissions within 12 months

Figure 2 Number of hospital admissions within 12 months in the control and intervention group.

this perspective to the body of evidence. We estimate the effect of the MR on direct patient outcomes such as hospital admissions, which is reliable data thanks to Swedish personal numbers.

Another strength of this study is the long follow-up time (12 months), which is rare in this kind of study, ${ }^{19}$ due to the high mortality rate among this frail group of elderly patients. The two most common intervention recommendations the pharmacist presented to the physician were withdrawal of drug therapy and reduced dosage. Many PIMs, as long-acting benzodiazepines and tramadol need a longer withdrawal period with an initial stepwise lowered dosage. The long follow-up period in the present study allowed a clinical effect of the medication withdrawal; this is a strength of this study. However, a major limitation is that additional data about medications were not collected after the initial follow-up at two months from base line, to confirm the assumption of maintained or increased withdrawal of PIMs. Data from the 2 months follow-up in the original RCT showed that $56 \%$ of the suggested medication changes resulted in actions taken by the physician. ${ }^{11}$ The decision of medication changes was taken by the responsible physician based on the knowledge of the patient in combination with recommendations from the MR. Therefore, not all proposed changes were accepted by the physician based

Table 4 Mean Survival Time in the Control and Intervention Groups at 12 Months Follow-Up

\begin{tabular}{|l|l|l|l|l|l|l|}
\hline Intervention/Control & N & Minimum & Maximum & Mean & Std. Deviation \\
\hline \multirow{2}{*}{ Control } & Days to first hospital admission & 187 & 0 & 361 & 160.61 & 69.7 \\
& $\begin{array}{l}\text { Days to death } \\
\text { Valid N }\end{array}$ & 187 & 5 & 363 & 175.23 & 58.1 \\
& $\begin{array}{l}\text { Days to first hospital admission } \\
\text { Intervention }\end{array}$ & 182 & 4 & 356 & 167.04 & 54.4 \\
& Days to death & 182 & 2 & 341 & 175.40 & 56.1 \\
& Valid N & 182 & & & & \\
\hline
\end{tabular}




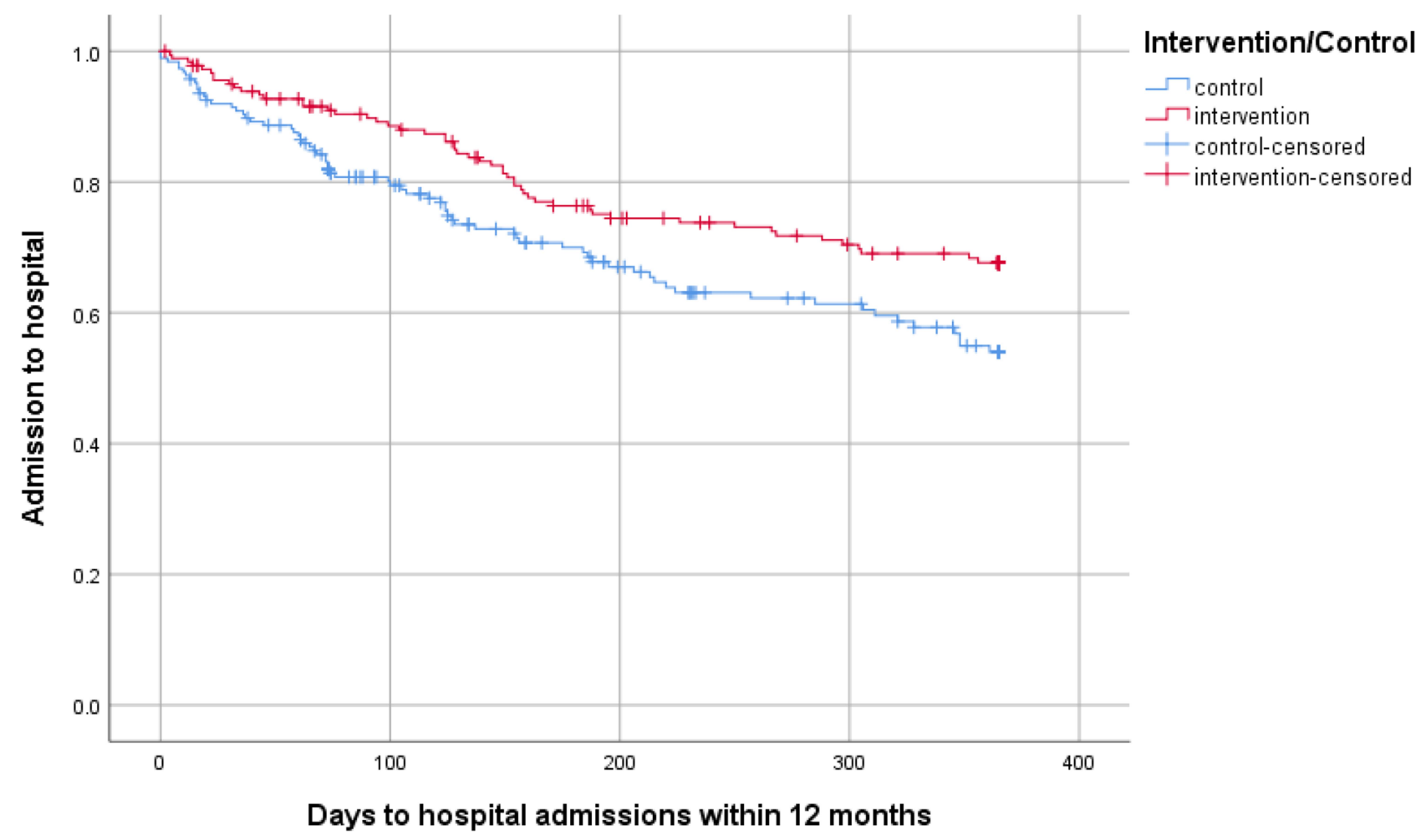

Figure 3 Output for Kaplan-Meier estimates for days to first hospital admission in intervention and control group at 12 months.

on what was considered most beneficial for the patient This may have affected our results, but we believe that MRs gave the physician a thorough view of the patients' medications that matters even if not all proposed changes were made.

Censoring the individuals in the control group, which had received a MR, when the survival analysis was performed is also a strength of the study.

A major limitation of the study is the lack of information about patients' base line comorbidities, the reason for hospital admissions or if the hospital admission was due to a DRP. Information about the cause of hospital admission was available but not conclusive, so we chose not to analyze it. Some of the hospital admissions could be assumed to be related to DRPs as the previous paper on the same population showed a high incidence of reported falls (29\%) and severe falls leading to hospital admissions (17\%) prior to baseline. ${ }^{11}$ Thus, further analysis on the possible association between DRPs and hospital admissions needs to be performed.

We have not analyzed data on morbidity such as number of chronic conditions and/or cognitive impairment, and this is a limitation of the study. Given the similarities in the number of drugs both between groups and between patients in different living forms, we can only assume that there were similar levels of multimorbidity in the groups.

We found a positive effect of MRs on the risk for hospital admissions. As it is not solely about avoiding hospital admission, the impact of MRs on quality of life would be interesting to assess as only a few studies have raised this important question. ${ }^{7,20}$

Our results showed that MRs had no effect on mortality or on time to death when the intervention group and control group were compared, which is in-line with other research. ${ }^{4,21}$

It has been argued that MRs are part of the physicians' professional responsibilities. However, the multi-professional approach with pharmacist and nurse involvement might bring different dimensions upon patients' medication list, giving additional attention to frail elderly in an over-burdened primary care.

This study included a group of elderly who represents the frailest and most care-needing population in the country. For a frail elderly person with multimorbidity, hospital admissions can contribute to further confusion and sometimes do more harm than good. Therefore, we suggest that frail elderly individuals 
should be treated with increased attention, including MRs, to reduce avoidable hospital admissions.

\section{Conclusion}

The results show that MRs can have a significant impact on the risk for hospital admissions but not on mortality rate. MRs should be considered in the care of frail elderly patients with expected benefits on avoiding hospital admissions.

\section{Abbreviations}

DRP, drug-related problem; EMR, electronic medical record; FRIDs, fall risk increasing drugs; GP, general practitioner; MR, medication review; $\mathrm{PHCC}$, primary healthcare center; PIM, potentially inappropriate medication; RCT, randomized controlled trial.

\section{Data Sharing Statement}

The datasets used during the current study are available from the corresponding author on reasonable request. The datasets used during the current study are also submitted to Dryad (doi:10.5061/dryad.wm37pvmjv).

\section{Ethics and Consent to Participate}

This study was conducted in accordance with the Declaration of Helsinki. The study has been approved by the Regional Ethical Review Board in Lund (Reg. no. 2018/8). Patients were included in the study after they provided written consent directly or through relatives in cases of severe cognitive impairment. The study adheres to CONSORT and STROBE guidelines.

\section{Consent to Publish}

We have obtained informed and written consent to publish from all study participants, as required by the Regional Ethical regulations in Sweden.

\section{Acknowledgments}

We are indebted to Patrick Reilly for his expertise and invaluable advice in editing the manuscript, Sara Larsson Lönn and Ulf Jakobsson for statistical input.

\section{Author Contributions}

All authors made a significant contribution to the work reported, whether that is in the conception, study design, execution, acquisition of data, analysis and interpretation, or in all these areas; took part in drafting, revising or critically reviewing the article; gave final approval of the version to be published; have agreed on the journal to which the article has been submitted; and agree to be accountable for all aspects of the work.

\section{Funding}

This study was funded by Lions Forskningsfond Skåne as well as Elsa Lundbergs and Greta Flerons fond.

\section{Disclosure}

Dr Veronica Milos Nymberg reports grants from Lions Forskningsfond Skåne, during the conduct of the study. The authors declare that they have no other potential conflicts of interest for this work.

\section{References}

1. Hajjar ER, Cafiero AC, Hanlon JT. Polypharmacy in elderly patients. Am J Geriatr Pharmacother. 2007;5(4):345-351. doi:10.1016/j. amjopharm.2007.12.002

2. Brulhart MI, Wermeille JP. Multidisciplinary medication review: evaluation of a pharmaceutical care model for nursing homes. Int $J$ Clin Pharm. 2011;33(3):549-557. doi:10.1007/s11096-011-9506-1

3. Hellstrom LM, Bondesson A, Hoglund P, et al. Impact of the Lund Integrated Medicines Management (LIMM) model on medication appropriateness and drug-related hospital revisits. Eur J Clin Pharmacol. 2011;67(7):741-752. doi:10.1007/s00228-010-0982-3

4. Holland R, Desborough J, Goodyer L, Hall S, Wright D, Loke YK. Does pharmacist-led medication review help to reduce hospital admissions and deaths in older people? A systematic review and meta-analysis. $\mathrm{Br} J$ Clin Pharmacol. 2008;65(3):303-316. doi:10.1111/j.1365-2125.2007.03071.x

5. Modig S, Holmdahl L, Bondesson A. Medication reviews in primary care in Sweden: importance of clinical pharmacists' recommendations on drug-related problems. Int J Clin Pharm. 2016;38(1):41-45. doi:10.1007/s11096-015-0189-x

6. Lenander C, Bondesson A, Viberg N, Jakobsson U, Beckman A, Midlov P. Effects of an intervention (SAKLAK) on prescription of potentially inappropriate medication in elderly patients. Fam Pract. 2017;34(2):213-218. doi:10.1093/fampra/cmw121

7. Renaudin P, Boyer L, Esteve MA, Bertault-Peres P, Auquier P, Honore S. Do pharmacist-led medication reviews in hospitals help reduce hospital readmissions? A systematic review and meta-analysis. $\mathrm{Br} \quad \mathrm{J}$ Clin Pharmacol. 2016;82(6):1660-1673. doi:10.1111/bcp. 13085

8. Khalil H, Bell B, Chambers H, Sheikh A, Avery AJ. Professional, structural and organisational interventions in primary care for reducing medication errors. Cochrane Database Syst Rev. 2017;10: CD003942. doi:10.1002/14651858.CD003942.pub3

9. Odesjo H, Anell A, Boman A, et al. Pay for performance associated with increased volume of medication reviews but not with less inappropriate use of medications among the elderly - an observational study. Scand J Prim Health Care. 2017;35(3):271-278. doi:10.1080/ 02813432.2017.1358434

10. Midlov P, Holmdahl L, Eriksson T, et al. Medication report reduces number of medication errors when elderly patients are discharged from hospital. Pharm World Sci. 2008;30(1):92-98. doi:10.1007/ s11096-007-9149-4

11. Milos V, Rekman E, Bondesson A, et al. Improving the quality of pharmacotherapy in elderly primary care patients through medication reviews: a randomised controlled study. Drugs Aging. 2013;30 (4):235-246. doi:10.1007/s40266-013-0057-0 
12. Indikatorer för god läkemedelsterapi hos äldre. The Swedish National Board of Health and Welfare. Published 2010 2017. Available from: https:/www.socialstyrelsen.se/globalassets/sharepoint-dokument/arti kelkatalog/ovrigt/2010-6-29.pdf. Accessed september 24, 2020.

13. Oscanoa TJ, Lizaraso F, Carvajal A. Hospital admissions due to adverse drug reactions in the elderly. A meta-analysis. Eur J Clin Pharmacol. 2017;73(6):759-770. doi:10.1007/s00228-017-2225-3

14. Davies EC, Green CF, Mottram DR, Rowe PH, Pirmohamed M. Emergency re-admissions to hospital due to adverse drug reactions within 1 year of the index admission. Br J Clin Pharmacol. 2010;70 (5):749-755. doi:10.1111/j.1365-2125.2010.03751.x

15. Pirmohamed M, James S, Meakin S, et al. Adverse drug reactions as cause of admission to hospital: prospective analysis of 18820 patients. BMJ. 2004;329(7456):15-19. doi:10.1136/bmj.329.7456.15

16. Gillespie U, Alassaad A, Henrohn D, et al. A comprehensive pharmacist intervention to reduce morbidity in patients 80 years or older: a randomized controlled trial. Arch Intern Med. 2009;169 (9):894-900. doi:10.1001/archinternmed.2009.71

17. Gustafsson M, Sjolander M, Pfister B, Jonsson J, Schneede J, Lovheim H. Pharmacist participation in hospital ward teams and hospital readmission rates among people with dementia: a randomized controlled trial. Eur J Clin Pharmacol. 2017;73 (7):827-835. doi:10.1007/s00228-017-2249-8
18. Ravn-Nielsen LV, Duckert ML, Lund ML, et al. Effect of an in-hospital multifaceted clinical pharmacist intervention on the risk of readmission: a randomized clinical trial. JAMA Intern Med. 2018;178(3):375-382. doi:10.1001/jamainternmed.2017.8274

19. Huiskes VJ, Burger DM, van den Ende $\mathrm{CH}$, van den Bemt BJ. Effectiveness of medication review: a systematic review and meta-analysis of randomized controlled trials. BMC Fam Pract. 2017;18(1):5. doi:10.1186/s12875-016-0577-x

20. Lenander C, Elfsson B, Danielsson B, Midlov P, Hasselstrom J. Effects of a pharmacist-led structured medication review in primary care on drug-related problems and hospital admission rates: a randomized controlled trial. Scand J Prim Health Care. 2014;32 (4):180-186. doi:10.3109/02813432.2014.972062

21. Wallerstedt SM, Kindblom JM, Nylen K, Samuelsson O, Strandell A. Medication reviews for nursing home residents to reduce mortality and hospitalization: systematic review and meta-analysis. $\mathrm{Br} \mathrm{J} \mathrm{Clin}$ Pharmacol. 2014;78(3):488-497. doi:10.1111/bcp.12351
Drug, Healthcare and Patient Safety

\section{Publish your work in this journal}

Drug, Healthcare and Patient Safety is an international, peer-reviewed open-access journal exploring patient safety issues in the healthcare continuum from diagnostic and screening interventions through to treatment, drug therapy and surgery. The journal is characterized by the rapid reporting of reviews, original research, clinical, epidemiological and post-marketing surveillance studies, risk management, health

\section{Dovepress}

literacy and educational programs across all areas of healthcare delivery. The manuscript management system is completely online and includes a very quick and fair peer-review system. Visit http://www.dovepress.com/testimonials.php to read real quotes from published authors. 